Article

\title{
Effect of Gradation Segregation on Mechanical Properties of an Asphalt Mixture
}

\author{
Wenliang Wu ${ }^{1}$, Zhixian Tu ${ }^{1}$, Zihan Zhu ${ }^{1} \mathbb{D}$, Zeyu Zhang ${ }^{2, *} \mathbb{D}$ and Yongjie Lin ${ }^{1, *}$ \\ 1 School of Civil Engineering and Transportation, South China University of Technology, Guangzhou 510000, \\ China; ctwlwu@scut.edu.cn (W.W.); 201620106350@mail.scut.edu.cn (Z.T.); \\ 201730187102@mail.scut.edu.cn (Z.Z.) \\ 2 Institute of Highway Engineering, RWTH Aachen University, 52066 Aachen, Germany \\ * Correspondence: zeyu.zhang@isac.rwth-aachen.de (Z.Z.); linyjscut@scut.edu.cn (Y.L.)
}

Received: 7 December 2018; Accepted: 11 January 2019; Published: 16 January 2019

\begin{abstract}
Gradation segregation strongly affects the service performance of asphalt pavement. However, rare studies focus on the influences of segregation on asphalt mixture as it is difficult to simulate segregation in a laboratory. This paper presents a research aiming to evaluate the effects of gradation segregation using the two dimensional (2D) discrete element method (DEM). To achieve this objective, the gradation segregation levels are defined according to the differences between control and segregated gradations. Based on this, asphalt mixtures with three gradations at three segregation levels were selected. The corresponding 2D DEM models were then built using Particle Flow Code 2D (PFC 2D). The aggregate skeletons in the asphalt mixture were determined separately by means of the contact point and contact force. Based on this, the variations on the aggregate skeleton, aggregate distribution, and mechanical properties of the asphalt mixture under vertical load were studied at three different segregation levels. This study indicates that the aggregate skeleton in asphalt mixture can be affected by the gradation segregation. The correlation between gradation segregation and mechanical property is also determined in this study.
\end{abstract}

Keywords: asphalt mixture; gradation segregation; aggregate skeleton; discrete element method

\section{Introduction}

Gradation segregation is defined as the non-uniform distribution of coarse and fine aggregates in paved asphalt pavement [1]. It may occur during plant manufacturing, truck transportation, and paving of asphalt mixtures. Localized areas rich in coarse and fine aggregates are termed as coarse and fine aggregate segregation, respectively. Gradation segregation is one of the main factors affecting the durability of asphalt pavement, because both coarse and fine aggregate segregation may lead to pavement failures. These failures reduce the service performance and service life of asphalt pavement [2-5]. Therefore, efforts have been devoted by various researchers for gaining improved insight into the mechanism, affecting factors, measurement and evaluation methods of aggregate gradation segregation [6-8]. Digital image processing techniques, non-nuclear densitometers, infrared camera, and industrial x-ray computed tomography (CT) have been used to study the segregation within asphalt mixtures during transportation and paving. Bruno L et al. [9] used an image analysis method to finalize an effective analysis of asphalt section image for automatically extracting aggregate gradation without the need of separating the bitumen from the aggregate. Wangheng J et al. [10] investigated the spreading segregation and compaction segregation of two asphalt mixtures by sieving and non-nuclear density gauge. Based on the findings, asphalt mixtures with spreading and compaction segregation were prepared in laboratory. The corresponding moisture stability was then evaluated in laboratory. Dajin G et al. [11] determined the feasibility of using infrared camera as the tool for 
analyzing the temperature segregation of asphalt mixture. Tao L et al. [12] presented an imaging method to assess the homogeneity of asphalt concrete using X-ray computed tomography, the improved OTSU image method, and fractal theory. The pavement-quality indicators were applied to test asphalt pavement and to choose four specimens with various degrees of segregation. Finally, a segregation evaluation standard based on fractal dimensions and the imaging method was proposed.

In addition, the effect of segregation on the performance of asphalt pavement and the corresponding quality control methods were also documented in many publications [13-15]. Based on previous studies, it was recommended that the effects of segregation and air voids should be taken into consideration when building the correlation between tensile strength and pavement performance. Besides, it is also found that the fatigue life and moisture resistance of asphalt mixture strongly related to the aggregate segregation. Mahoney JP et al. [16] used an infrared camera to identify cooler portions of the mat.the aggregate gradation and asphalt binder content of asphalt mixture was also determined. The results showed no significant aggregate segregation within the cooler areas; however, these cooler portions of the mat consistently showed higher air voids than the surrounding pavement. The influence of aggregate segregation on rutting resistance and raveling was also reported in previous studies [3]. Researchers also tried to determine factors affecting the gradation segregation, for instance, mixing plant, volumetric properties, aggregate shape properties, mixing temperature, paving conditions etc. [17-19]. However, the above studies have some limitations. Both laboratory and in field tests are conducted with phenomenological methods. Changes in the micro-parameters of asphalt mixture resulting from gradation segregation are unclear. Low repeatability of test results occurs because of various experimental variables. Despite this, the correlation between gradation segregation and mechanical property of asphalt mixture still deserves more effort.

DEM has been applied to analysis road material performance because of its superiority in address non-uniformity, discontinuity, and large deformation problems [20-23]. Zelelew HM et al. [20] using DEM to describe the microstructure of asphalt mixtures and simulate creep behavior. Coenen AR et al. [21] developed a software for processing and analyzing 2D images of asphalt mixtures. By means of this software, radial distribution, orientation and segregation of aggregates were analyzed. Wagoner MP et al. [22] described the development of a fracture test for determining the fracture energy of asphalt concrete. Masad E et al. [23] investigated the stiffness anisotropy of asphalt mixtures using micromechanics-based models. Therefore, this paper presents an attempt to evaluate the effects of gradation segregation on asphalt mixture in terms of structural characteristics and mechanical properties using the DEM method. To achieve this objective, a 2D asphalt mixture model was built using PFC2D5.0 software, which is a computing software developed by the Itasca company in the USA. Based on the 2D DEM model, the aggregate skeleton in asphalt mixture and internal mechanical characteristics of asphalt mixture was studied. Furthermore, the mechanical performance such as resistance, transfer and diffusion to load of asphalt mixtures at different segregation levels was studied and compared.

\section{DEM Model Development}

\subsection{Segregation Simulation}

As the aggregate gradation in asphalt mixture changes through segregation, the differences between the control aggregate gradation and the segregated aggregate gradation can be used to determine the segregation levels. In this research, the area between the segregated gradation curve and the control gradation curve was employed to quantify the segregation of aggregate in asphalt mixture. A large area indicates a high segregation level. As shown in Equation (1), $S$ value can be used to reflect the area difference between the segregated and the standard gradation curves [24].

$$
S=\sqrt{\sum_{i=1}^{\mathrm{n}}\left(p_{i j}-p_{a j}\right)^{2}}
$$


where,

$\mathrm{n}$ is different sieve size level,

$P_{i j}$ is the passing percentage of the segregated gradation, and

$P_{a j}$ is the passing percentage of the standard gradation.

Based on the calculated $S$ value, the gradation segregation can be divided into four levels: no segregation, low-level segregation, medium-level segregation, and high-level segregation with the corresponding $S$ value of less than $10 \%, 10-20 \%, 20-35 \%$, and greater than $35 \%$, respectively.

To evaluate the effect of segregation on the aggregate skeleton in asphalt mixture, three gradations and three segregation levels were considered in this study. Table 1 presents the labels of the involved aggregate gradations. Control aggregate gradation (no segregation) in this study is labelled as D.

Table 1. Label of aggregate gradations.

\begin{tabular}{cccc}
\hline & High-Level Segregation & Medium-Level Segregation & Low-Level Segregation \\
\hline Coarse gradation & $\mathrm{HC}$ & $\mathrm{MC}$ & $\mathrm{LC}$ \\
Fine gradation & $\mathrm{HF}$ & $\mathrm{MF}$ & $\mathrm{LF}$ \\
\hline
\end{tabular}

The $S$ value of HC, MC, LC, D, LF, MF, and HF is $37.4 \%, 21.9 \%, 11.3 \%, 0,10.7 \%, 21.7 \%$, and $38 \%$, respectively and the corresponding gradations are listed in Table 2.

Table 2. Composition of asphalt mixtures.

\begin{tabular}{cccccccc}
\hline \multirow{2}{*}{ Sieve Size/mm } & \multicolumn{7}{c}{ Passing Ratio (\%) for Different Sieving Size (mm) } \\
\cline { 2 - 8 } & $\boldsymbol{H F}$ & $\boldsymbol{M F}$ & $\boldsymbol{L F}$ & $\boldsymbol{D}$ & $\boldsymbol{L C}$ & $\boldsymbol{M C}$ & $\boldsymbol{H C}$ \\
\hline 26.5 & 100 & 100 & 100 & 100 & 100 & 100 & 100 \\
19 & 100 & 96 & 94 & 92 & 91 & 88 & 83 \\
16 & 97 & 88 & 84 & 81 & 76 & 74 & 66 \\
13.2 & 84 & 76 & 72 & 67 & 64 & 58 & 50 \\
9.5 & 73 & 66 & 60 & 55 & 48 & 44 & 37 \\
4.75 & 51 & 46 & 40 & 35 & 30 & 24 & 19 \\
2.36 & 36 & 30 & 27 & 23 & 20 & 16 & 11 \\
1.18 & 23 & 21 & 17 & 16 & 14 & 11 & 9 \\
0.6 & 16 & 14 & 13 & 11 & 9 & 8 & 6.5 \\
0.3 & 10.5 & 10 & 8 & 8 & 7 & 6 & 5.5 \\
0.15 & 8 & 7 & 6.5 & 6 & 5.5 & 5 & 4 \\
0.075 & 6 & 5.5 & 5 & 4.5 & 4 & 3 & 2.5 \\
Asphalt binder content (\%) & 5.4 & 4.9 & 4.5 & 4.1 & 3.7 & 3.3 & 2.8 \\
\hline
\end{tabular}

\subsection{Model Development}

To simplify the DEM model, aggregates in asphalt mixture are modeled as spheres with different diameters. In addition, it is assumed that the density of all the aggregates is same and the surface area of aggregates passing sieve is uniformly distributed. Therefore, the mass ratio of aggregate with different diameters equals their area percentage per unit area. The number of aggregate particles in asphalt mixture can be approximately calculated through Equation (2) to Equation (3):

$$
\begin{gathered}
A_{i}=A \times(1-\theta) \times P_{i} \\
n_{i}=\frac{A_{i}}{\pi \cdot\left(r_{\text {min }}^{2}+r_{\text {max }}^{2}\right) / 2}
\end{gathered}
$$

where,

$A=$ total area of the designed model;

$\Theta=$ voids in the mineral aggregate; 
$P_{i}=$ percentage of aggregate remaining on the $i$-th sieve;

$A_{i}=$ total area of aggregate remaining on the $i$-th sieve;

$n_{i}=$ total number of the aggregate remaining on the $i$-th sieve;

$r_{\max }=$ maximum radius of the spheres

$r_{\min }=$ minimum radius of the spheres.

To calculate the number of aggregates with different diameters, the air voids in mineral aggregate (VMA) should be calculated in advance. In this study, VMAs were calculated by means of trial-calculating. Specifically, let the model area equal the total area of aggregates. The amounts of aggregate with different diameters can be estimated. Then, develop the DEM model accordingly followed by calculating the VMA of aggregates within the built DEM model. For each gradation, the VMA calculation trial-calculating process was repeated 10 times to minimize errors. The trail-calculated VMAs are listed in Table 3.

Once the VMA was obtained, the amounts of aggregate spheres can be calculated according to Equations (2) and (3). However, due to the randomness of the generated particle area, in order to ensure that the total area of each aggregate gradations is consistent with the design value, after generating the aggregate particles for each gradation according to the calculated resluts, it is necessary to count the total area of all particles. Compared with the design value, if the difference between the two is greater than $\pi \cdot\left(r_{\min }^{2}+r_{\max }^{2}\right) / 2$, the result is modified by randomly adding or deleting one particle, and then repeat the above process until the area of each grade of aggregate particles meets the design requirement. Based on this, 2D DEM model is built.

Figure 1 shows the 2D DEM model development process. A $150 \times 100 \mathrm{~mm}$ size model was built firstly by generating four rigid walls. Then, loose aggregates were generated uniformly in the certain space surrounded by the four walls. A certain speed was then given to the upper and lower walls for compacting the loose aggregates. Finally, to avoid the aggregates being excessively compacted, the upper wall moves up a small distance, and then the PFC "solve" command was used to delete the imbalance force in the model. Repeat this step until the stress on the upper wall is 0 . The heights of the models can be found in Table 3. As excepted the heights of the models are basically consistent and this meets the research expectation.

Table 3. VMA of the mixture and height of the model.

\begin{tabular}{cccccccc}
\hline Gradation & $\boldsymbol{H C}$ & $\boldsymbol{M C}$ & $\boldsymbol{L C}$ & $\boldsymbol{D}$ & $\boldsymbol{L F}$ & $\boldsymbol{M F}$ & $\boldsymbol{H F}$ \\
\hline VMA/\% & 13.77 & 12.03 & 11.01 & 10.45 & 9.98 & 9.56 & 9.17 \\
Model height/mm & 100.2 & 100.5 & 100.3 & 100.9 & 100.4 & 100.7 & 100.1 \\
\hline
\end{tabular}

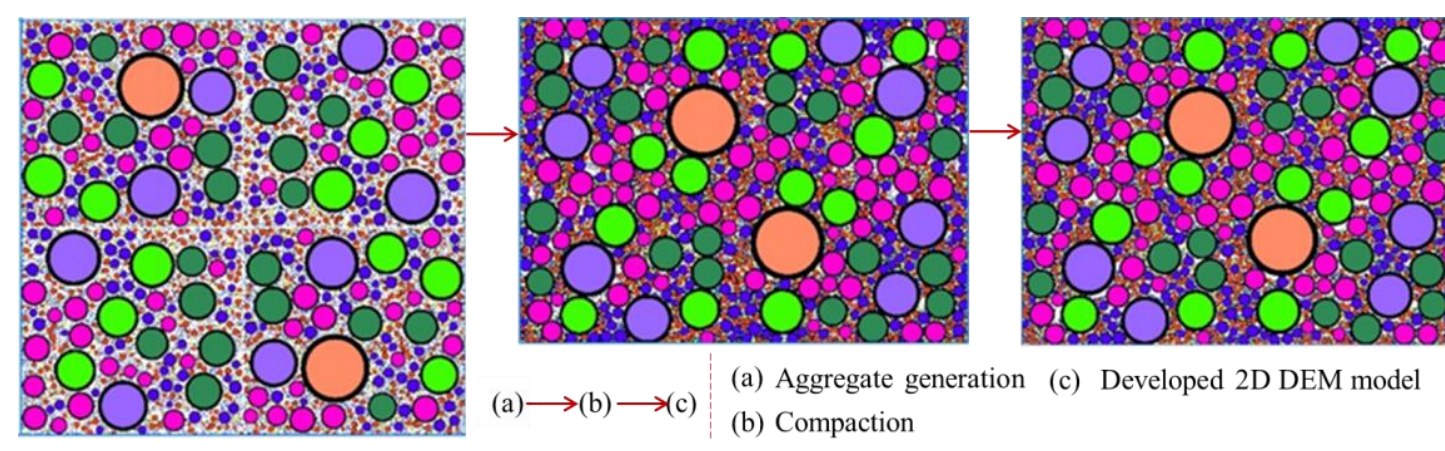

Figure 1. Development of 2D DEM Model.

\subsection{Contact Model and Model Parameters}

In order to describe the interaction amongst the aggregates in asphalt mixture, two bonding models are available in $\mathrm{PFC}^{2 \mathrm{D}}$, contact-bond model and parallel-bond model. The main difference 
between these two bond models is that the contact-bond model can only transmit force through the contact point, whereas the parallel-bond provides possibility for both transmitting force and moment. In addition, the parallel-bond model also adds the effect of binding materials [25]. Thus, the parallel-bond model was employed in this study to simulate the mechanical response of the aggregate skeleton in asphalt mixtures. The parallel-bond model can be viewed as the combination of serval springs with normal and tangential stiffness.

The micro-parameters for developing the asphalt mixture model were collected from laboratory test, i.e. uniaxial compression test. The test was performed by the US MTS810 material test system with the test temperature and load speed of $20^{\circ} \mathrm{C}$ and $1 \mathrm{~mm} / \mathrm{s}$, respectively. Vertical loadings were conducted on the DEM model and laboratory prepared asphalt samples. When the vertical deformation of asphalt mixture sample arrives at $\Delta h$, the corresponding vertical load in the laboratory and DEM model is recorded and labelled as $P_{i}$ and $P_{i}{ }^{\prime}$, respectively. The difference between $P_{i}$ and $P i^{\prime}$ is defined by $\beta_{i}=\left|P_{i}-P_{i}^{\prime}\right| / P_{i}$. Micro-parameters were adjusted for minimizing $\beta$. The obtained micro-parameters used in the DEM model are presented in Table 4. The mechanical response of asphalt mixture samples and the corresponding DEM models under vertical load are presented in Table 5. As can be seen, the difference in loading between the DEM model and laboratory test is less than $8 \%$, indicating that the microscopic parameters obtained by this method are appropriate.

Table 4. Microscopic parameters for contact of the skeleton model.

\begin{tabular}{ccc}
\hline Model Component & Microscopic Parameters & Value \\
\hline \multirow{2}{*}{ Spheres } & Density $\rho /\left(\mathrm{kg} \cdot \mathrm{m}^{-3}\right)$ & 2600 \\
& Friction coefficient $\mu$ & 0.5 \\
& Normal stiffness $K_{n} /\left(\mathrm{N} \cdot \mathrm{m}^{-1}\right)$ & $5.2 \times 10^{8}$ \\
& Shear stiffness $K_{s} /\left(\mathrm{N} \cdot \mathrm{m}^{-1}\right)$ & $5.2 \times 10^{8}$ \\
& Normal parallel-bond stiffness $p b \_k_{n} /\left(\mathrm{N} \cdot \mathrm{m}^{-1}\right)$ & $1.1 \times 10^{5}$ \\
& Shear parallel-bond stiffness $p b \_k_{s} /\left(\mathrm{N} \cdot \mathrm{m}^{-1}\right)$ & $1.1 \times 10^{5}$ \\
Boundary wall & Bond radius $r$ & 0.5 \\
& Friction coefficient $\mu_{w}$ & 0 \\
& Normal stiffness $K_{w n} /\left(\mathrm{N} \cdot \mathrm{m}^{-1}\right)$ & $2.3 \times 10^{8}$ \\
Other & Shear stiffness $K_{w s} /\left(\mathrm{N} \cdot \mathrm{m}^{-1}\right)$ & $2.3 \times 10^{8}$ \\
\hline
\end{tabular}

Table 5. Vertical loading in laboratory and DEM model.

\begin{tabular}{|c|c|c|c|c|c|c|c|c|c|c|}
\hline \multirow{2}{*}{\multicolumn{2}{|c|}{$\begin{array}{c}\text { Gradation } \\
\text { Load }\end{array}$}} & \multicolumn{3}{|c|}{$H C$} & \multicolumn{3}{|c|}{$M C$} & \multicolumn{3}{|c|}{$L C$} \\
\hline & & $P / \mathrm{MPa}$ & $P^{\prime} / \mathrm{MPa}$ & $\beta / \%$ & $\mathrm{P} / \mathrm{MPa}$ & $P^{\prime} / \mathrm{MPa}$ & $\beta / \%$ & $\mathrm{P} / \mathrm{MPa}$ & $P^{\prime} / \mathrm{MPa}$ & $\beta / \%$ \\
\hline \multirow{3}{*}{$\Delta h / \mathrm{mm}$} & 2 & 2.12 & 2.03 & 4.57 & 2.27 & 2.19 & 3.88 & 2.36 & 2.26 & 4.23 \\
\hline & 4 & 4.74 & 4.63 & 2.45 & 5.55 & 5.41 & 2.56 & 5.75 & 5.57 & 3.18 \\
\hline & 6 & 7.93 & 7.78 & 1.94 & 9.71 & 9.51 & 2.11 & 10.02 & 9.75 & 2.78 \\
\hline \multicolumn{2}{|c|}{ Gradation } & \multicolumn{3}{|c|}{$D$} & \multicolumn{3}{|c|}{$L F$} & \multicolumn{3}{|c|}{$M F$} \\
\hline \multicolumn{2}{|c|}{ Load } & $\mathrm{P} / \mathrm{MPa}$ & $P^{\prime} / \mathrm{MPa}$ & $\beta / \%$ & $\mathrm{P} / \mathrm{MPa}$ & $P^{\prime} / \mathrm{MPa}$ & $\beta / \%$ & $\mathrm{P} / \mathrm{MPa}$ & $P^{\prime} / \mathrm{MPa}$ & $\beta / \%$ \\
\hline \multirow{3}{*}{$\Delta h / \mathrm{mm}$} & 2 & 2.70 & 2.56 & 5.65 & 3.06 & 2.89 & 6.01 & 3.21 & 3.04 & 5.46 \\
\hline & 4 & 6.67 & 6.36 & 4.87 & 7.52 & 7.28 & 3.24 & 7.92 & 7.62 & 3.89 \\
\hline & 6 & 11.14 & 10.80 & 3.12 & 13.19 & 12.83 & 2.79 & 13.73 & 13.33 & 3.01 \\
\hline \multicolumn{2}{|c|}{ Gradation } & & $H F$ & & & & & & & \\
\hline \multicolumn{2}{|c|}{ Load } & $P / \mathrm{MPa}$ & $P^{\prime} / \mathrm{MPa}$ & $\beta / \%$ & & & & & & \\
\hline \multirow{3}{*}{$\Delta h / \mathrm{mm}$} & 2 & 3.35 & 3.11 & 7.71 & & & & & & \\
\hline & 4 & 8.16 & 7.77 & 5.02 & & & & & & \\
\hline & 6 & 14.25 & 13.62 & 4.65 & & & & & & \\
\hline
\end{tabular}




\section{Results}

\subsection{Effect of Gradation Segregation on the Aggregate Skeleton}

Aggregate skeleton plays a critical role in terms of asphalt mixture's service performance [26,27]. In this study, aggregates composing the aggregate skeleton in asphalt mixture were determined according to their role under loading. Figure 2 presents the contact points and contact forces of D-type asphalt mixture with the deformation of $2 \mathrm{~mm}$. In Figure 2a, dark and light gray spheres indicate aggregates with diameters greater and less than $2.36 \mathrm{~mm}$, respectively. Green dots between two simplified aggregate spheres are contact points. Black lines with different widens inside spheres representing contact force with magnitudes.

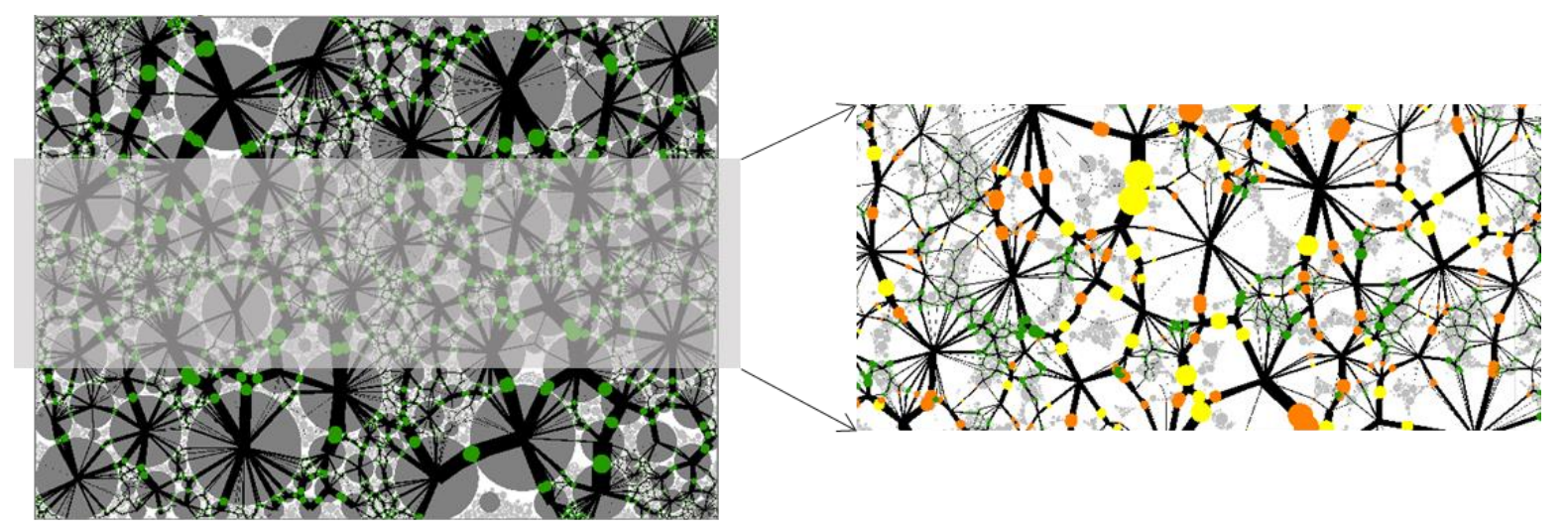

(a)

(b)

Figure 2. Distribution of contact points and contact force at larger (a) and smaller (b) scales.

Based on the data presented in Figure 2a, it can be seen that vertical load is transferred through aggregates with diameters larger than $2.36 \mathrm{~mm}$. Thus, the aggregate in the asphalt mixture with a diameter larger than $2.36 \mathrm{~mm}$ consists of the aggregate skeleton and the remaining fine aggregates mainly play a role of filling. In Figure $2 b$, green dots indicate the contact points between aggregates with diameter less than $2.36 \mathrm{~mm}$, yellow dots indicate contact points between the aggregates with diameter greater than $2.36 \mathrm{~mm}$, and the orange dots indicate the contact points (transition contact points) formed due to aggregate $(<2.36 \mathrm{~mm})$ sandwiched by two aggregates $(>2.36 \mathrm{~mm})$. As shown in the figure, the contact points on the main force chains are basically the yellow contact points and the orange contact points, while the other contact points (green) are hardly on the main force chain. This demonstrates that the skeleton of mixture mainly consists of aggregates with diameters greater than $2.36 \mathrm{~mm}$.

To further verify the above conclusions, according to the particle size of the aggregates at both ends of the contact, the contacts between the aggregates are divided into four types: blue, red, black and green, as shown in Figure 3a. The maximum, minimum and average values of the forces of four kinds of contact are shown in Table 6. Aggregates with a particle size greater than $2.36 \mathrm{~mm}$ are defined as coarse aggregates, and aggregates with a particle size less than $2.36 \mathrm{~mm}$ are defined as fine aggregates. It can be seen from Table 6 that the contact force at the blue contact is about 8 times that of the black contact, which means that the contact force between the coarse aggregates is much larger than the contact force between the fine aggregates. When the fine aggregates are in contact with the coarser aggregate, although the contact force increases, the value is still small. When the fine aggregates are sandwiched between two coarse aggregates, the value of the contact force is large, which is also due to the interaction between the coarse aggregates. Therefore, it can be concluded that in the D-type mixture, the coarse aggregates interact to form a skeleton which plays a major role in resisting external loads. 


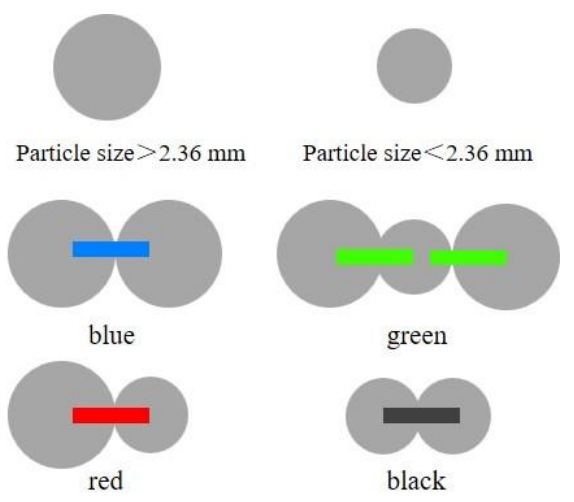

(a) contact types

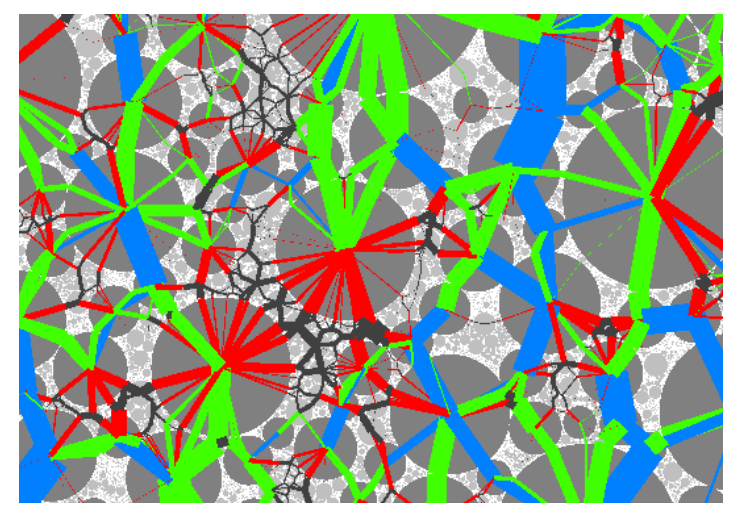

(b) contact force

Figure 3. Distribution of contact force.

Table 6. The contact force of four contact types.

\begin{tabular}{ccccc}
\hline \multirow{2}{*}{ Contact Force/N } & \multicolumn{4}{c}{ The Contact Types } \\
\cline { 2 - 5 } & Blue & Green & Red & Black \\
\hline maximum values & 35203 & 28837 & 13738 & 14471 \\
minimum values & 154 & 0.15 & 0.003 & $2.01 \times 10^{-6}$ \\
average values & 10079 & 6162 & 2098 & 1340 \\
\hline
\end{tabular}

In order to further analyze the impact of gradation segregation on the aggregate skeleton in the asphalt mixture, the HC and HF-types were studied as example. Figure 4 illustrates the distribution of contact points and contact stress. In Figure $4 \mathrm{a}$, the dark and light grey disks represent aggregates with diameter greater and less than $4.75 \mathrm{~mm}$, respectively. While, in Figure $4 \mathrm{~b}$, the dark and light grey disks represent aggregates with diameter greater and less than $1.18 \mathrm{~mm}$, respectively. In both figures, green dots represent the contact points between aggregates. Combining Figures 2 and 4, it is easy to see that the gradation segregation significantly affects the aggregate skeleton in the asphalt mixture. Specifically, with the increase of segregation, the aggregate skeleton in the asphalt mixture changes accordingly. Given this, it can be proposed that the mechanical properties of the asphalt mixture can be affected by the gradation segregation as the aggregate skeleton undergoes the main load.

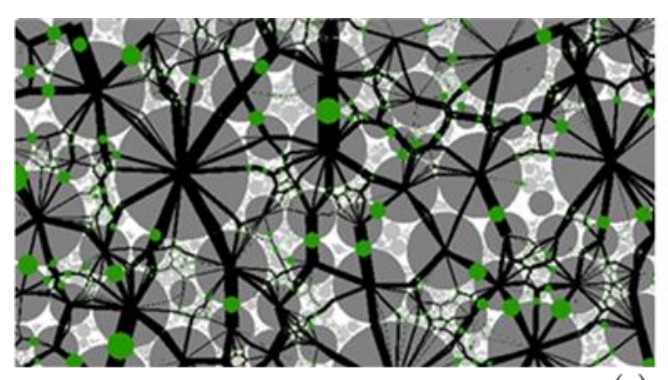

(a)

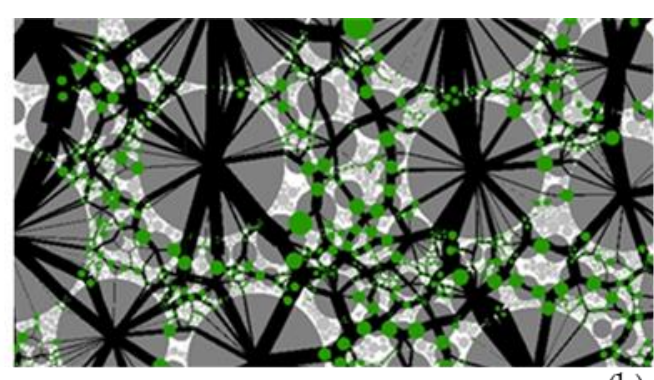

(b)

Figure 4. Distribution of contact points and contact force with different segregation levels: HC (a) and $\mathrm{HC}(\mathbf{b})$.

\subsection{Effect of Gradation Segregation on the Asphalt Mixture}

\subsubsection{Mechanical Characteristics of the Asphalt Mixture}

In this study, VMA, the number of contact points between the aggregates and the contact force at the contact points are involved as the mechanical characteristics of asphalt mixture. Based upon these 
parameters, the influence of gradation segregation on the mechanical properties of the asphalt mixture is analyzed and compared. The VMA and contact points are listed in Table 7.

Table 7. Mechanical characteristics of asphalt mixtures.

\begin{tabular}{ccccc}
\hline Gradation & VMA/\% & $\begin{array}{c}\text { Number of } \\
\text { Contact Points }\end{array}$ & $\begin{array}{c}\text { Average Contact } \\
\text { Force/kN }\end{array}$ & $\begin{array}{c}\text { Maximum Contact } \\
\text { Force/kN }\end{array}$ \\
\hline HC & 13.77 & 1980 & 4.24 & 43.63 \\
MC & 12.03 & 3061 & 2.85 & 34.55 \\
LC & 11.01 & 3514 & 2.21 & 28.72 \\
D & 10.45 & 6734 & 1.55 & 22.63 \\
LF & 9.98 & 7837 & 1.17 & 17.65 \\
MF & 9.56 & 13,054 & 0.98 & 14.56 \\
HF & 9.17 & 15,634 & 0.81 & 11.22 \\
\hline
\end{tabular}

After the model was established, a certain initial speed was given to the upper wall to make it load downwards, and the servo control system was used to control the load process until the loading stress was maintained at $2.5 \mathrm{MPa}$. After that, the PFC2D 5.0 subroutine was used to calculate the VMA, the total number of contact points, the average contact force, and the maximum contact force. The results are shown in Table 7.

In Table 7, it can be seen that with decreasing gradation segregation level, the VMA gradually decreases and the number of contact points increases dramatically.

To further analyze the change in the contact points, the aggregate was divided into three grades according to the particle size: $>13.2 \mathrm{~mm}, 2.36 \sim 13.2 \mathrm{~mm}$, and $<2.36 \mathrm{~mm}$. The number of contact points around every grade of aggregate particles was counted, as shown in Figure 5. It can be seen that with the gradation from coarse to fine, although there are vibrations on the number of contact points around the aggregates with diameters larger than $13.2 \mathrm{~mm}$, there is no obvious difference. Conversely, it should be noted that the number of contact points around aggregates with the diameter of $2.36 \sim 13.2 \mathrm{~mm}$ and smaller than $2.36 \mathrm{~mm}$ show continually growing trend. This indicates that with the increase of fine aggregates in the mixture, the contact points around the fine aggregates increase sharply, while the contact points around the coarser aggregates change slightly.
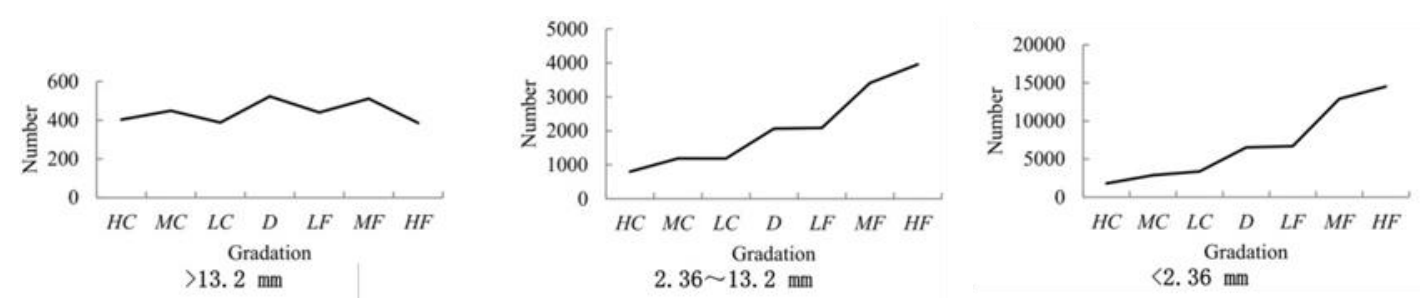

Figure 5. Change in the number of contact points.

When the loading stress was maintained at $2.5 \mathrm{MPa}$, the contact force distributing in the mixture was shown in Figure 6. In this figure, the thicker the black lines, the greater the contact forces. It can be seen from Figure 6 and Table 7 that with the gradation from coarse to fine, the contact force chains in the mixture gradually become thinner. It reveals that the average contact force and the maximum contact force gradually decrease. The average contact force of the HC-type mixture is about 5 times of that of the HF-type, and the maximum contact force is about 4 times.. Since aggregates are more susceptible to be crushed under high stress, when coarse aggregate segregation occurs, coarse aggregates in the mixture are more likely to be crushed under the action of vehicle loads, directly affecting the stability of skeleton structure and further affecting the road performance of the mixture. 


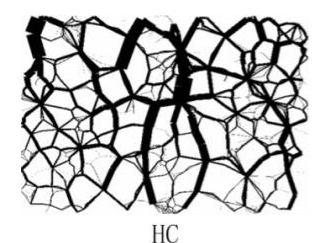

$\mathrm{HC}$

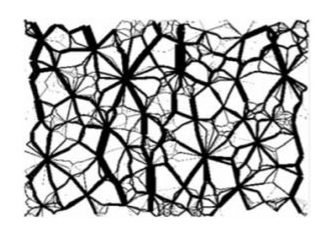

MC

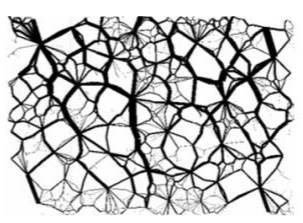

LC

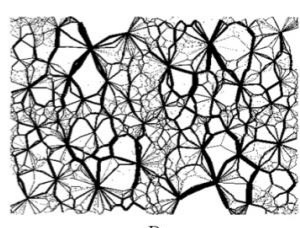

D

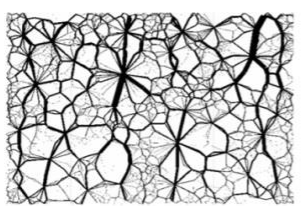

LF

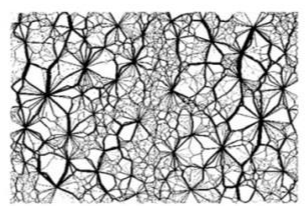

MF

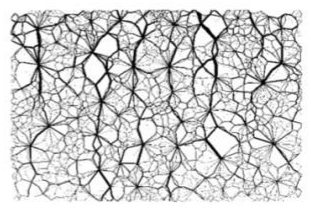

$\mathrm{HF}$

Figure 6. Distribution of contact force in asphalt mixtures.

\subsubsection{Compressive Strength}

In order to study the effect of gradation segregation on the performance of asphalt mixtures under compressive load, $10 \times 10 \mathrm{~cm}$ 2D DEM models were developed based on the method described above. The top wall was controlled to load down at a constant speed of $2 \mathrm{~mm} / \mathrm{min}$ until the model was broken. During this period, the stress on the top wall and the model strain were recorded, as shown in Figure 7. The compressive strength ratios of the seven kinds of mixtures were calculated, as shown in Figure 8 . The compressive strength ratio is defined as the compressive strength of segregation mixtures divided by the compressive strength of standard mixture.

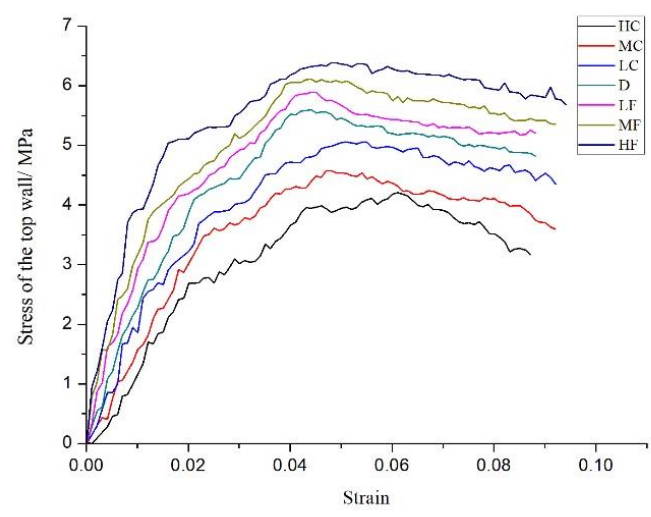

Figure 7. The stress-strain curves of the asphalt mixture.

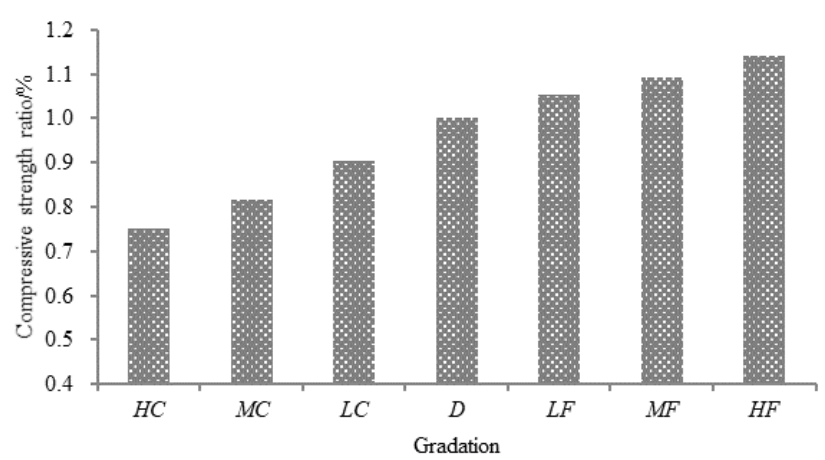

Figure 8. Compressive strength ratio of the asphalt mixture.

From Figures 7 and 8, it can be seen that with the gradation from coarse to fine, the compressive strength of mixture gradually increases. When the fine aggregate segregation occurs, the compressive strength of mixture increases with the increase of segregation levels with the increase rate of $5 \sim 14 \%$. While, when the coarse aggregate segregation occurs, the compressive strength of mixture significantly 
decreases with the increase of segregation level. Under high-level coarse aggregate segregation conditions, the compressive strength of mixture is about $75 \%$ of that of the standard mixture. Even under low-level coarse aggregate segregation, the compressive strength of mixture is only about $90 \%$ of that of the standard mixture. This indicates that the increase of fine aggregates in the mixture is beneficial to improve the compressive strength of the mixture, while if coarse aggregate segregation occurs in asphalt mixture, the compressive strength will decrease significantly with the increase of segregation level, which in turn increase the broken susceptibility of asphalt mixture under loading.

In order to verify the reliability of the simulation results, compression test was performed on asphalt mixture with MTS. The laboratory test parameters are same as that of the simulation. Figure 9 presents the differences between simulation and laboratory results. It can be found that the simulation results are almost consistent with the laboratory experiment results, which verified the feasibility of using the DEM to evaluate the anti-compression performance of the asphalt mixture.

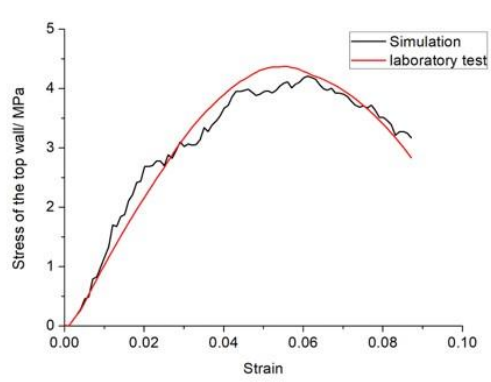

$\mathrm{HC}$

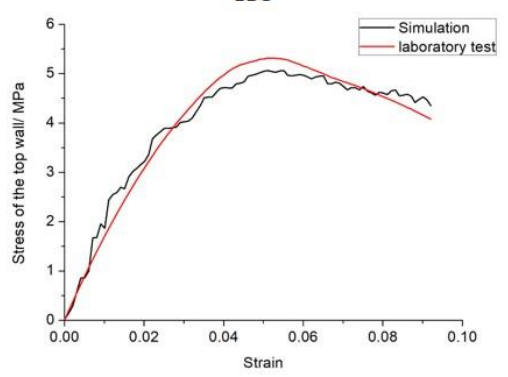

LC

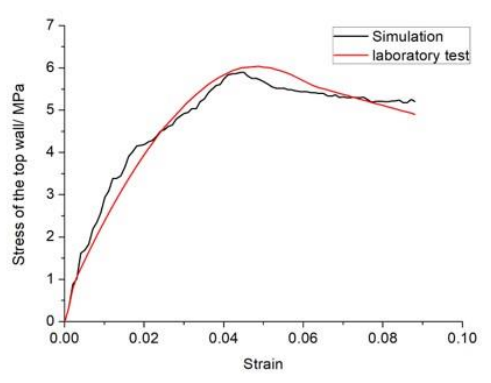

LF

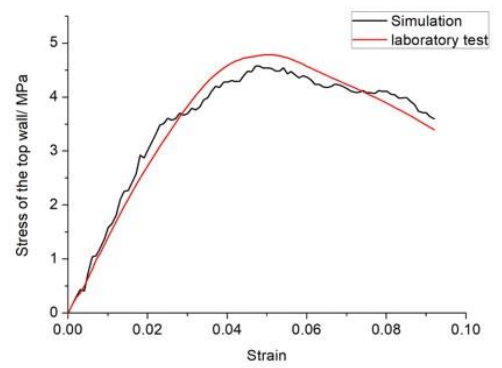

$\mathrm{MC}$

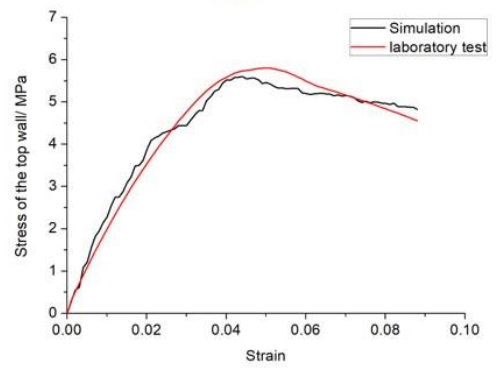

$\mathrm{D}$

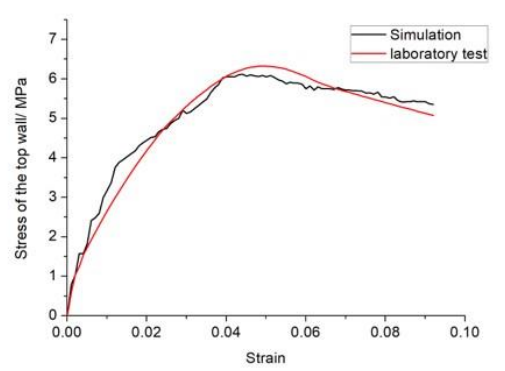

MF

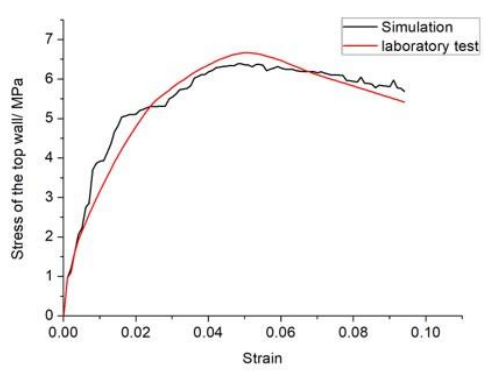

$\mathrm{HF}$

Figure 9. The stress-strain curves of the asphalt mixture. 


\subsubsection{Vertical Stress Transfer Performance}

In order to investigate the vertical stress transfer performance (VSTP) of the asphalt mixture at different segregation levels, the discrete element model was established according to the method described in 1.2. The width of the upper wall was set to $22 \mathrm{~cm}$ to better simulate the actual situation where the wheel load acts on the road surface. The heights of the model were taken as 4,6 and $8 \mathrm{~cm}$ for comparative analysis.

After the model was established, the upper wall was given a velocity of $0.1 \mathrm{~mm} / \mathrm{s}$ to load downwards until the vertical deformation of mixture reached $2 \mathrm{~mm}$. After finishing load, the average stress $\left(\overline{\sigma_{t}}\right)$ of the upper wall and the average stress $\left(\overline{\sigma_{d}}\right)$ of the bottom wall were measured, and their ratio $(\delta)$ was calculated. The VSTP is analyzed by comparing the difference of $\delta$ values. The result is shown in Figure 10.

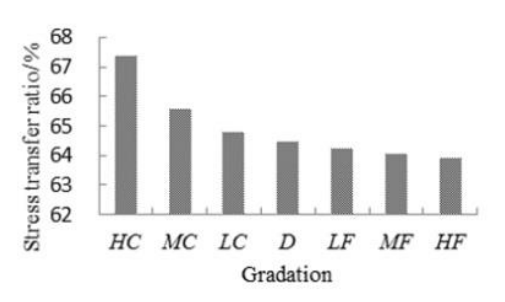

(a) $30 \times 4 \mathrm{~cm}$

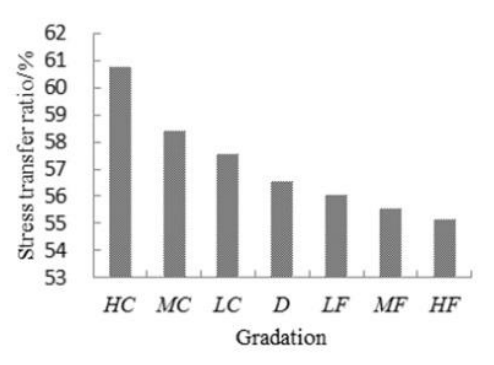

(b) $30 \times 6 \mathrm{~cm}$

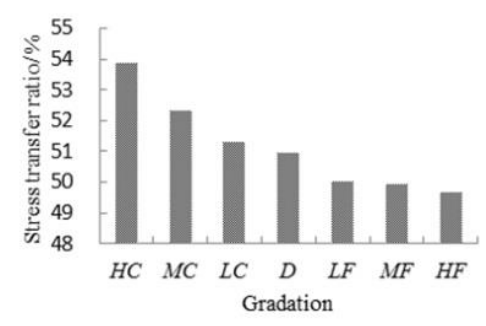

(c) $30 \times 8 \mathrm{~cm}$

Figure 10. VSTP of asphalt mixtures at different segregation levels.

Figure 10 shows the effect of gradation segregation and model dimensions on the VSTP of asphalt mixture. The data presented in Figure 10 illustrate that with the gradation from coarse to fine, the VSTP of asphalt mixture gradually decreases. In addition, when segregation of coarse aggregates occurs, the VSTP of the asphalt mixture is significantly improved. It is interesting to note that the increase rate raises results from the increase of segregation level. When segregation of fine aggregates occurs, the VSTP of the asphalt mixture has a slight decline and the difference between asphalt mixtures at different segregation levels is not significant. This indicates that the segregation of coarse aggregates has a greater impact on the VSTP of the mixture than the segregation of fine aggregates. In addition, as the height of the model increases, the vertical transfer distance of stress increases and the VSTP of asphalt mixture gradually weakens.

\subsubsection{Lateral Stress Diffusion}

In this study, the internal contact force and contact point distribution of the $30 \times 6 \mathrm{~cm}$ asphalt skeleton models of the HC, D, and HF are analyzed as examples. As shown in Figure 11, the HC-type mixture can uniformly diffuse the upper load through the skeleton to the bottom wall, while the upper load is more diffused in the middle part of the D-type and HF-type mixture skeletons and finally it mainly acts on the middle part of the bottom wall.

In order to further analyze the lateral stress diffusion performance (LSDP) of mixture skeletons at different segregation levels, after the finishing load, the stress on the middle $1 / 3$ section of the bottom wall and the stress on the total bottom wall and the corresponding ratio $\lambda$ were calculated. The result is shown in Figure 12.

Figure 12 shows that when segregation of coarse aggregates occurs in mixture, the $\lambda$ value is lower than that of the standard gradation mixture, but there was no obvious correlation between the $\lambda$ value and the degree of segregation; when segregation of fine aggregates occurs in mixture, the $\lambda$ value is higher than that of the standard gradation mixture, and the value of $\lambda$ gradually increases as the degree of segregation of fine aggregate intensifies. This further indicates that the mixture skeletons at a coarse aggregate segregation level can more evenly diffuse the stress to the bottom wall, and its LSDP 
is better. Further, the LSDP has no obvious correlation with the degree of segregation, while the stress concentration phenomenon is more likely to occur in the process of stress diffusion of the mixture skeletons at fine aggregate segregation level, and its LSDP is worse. As the degree of segregation of fine aggregates intensifies, the LSDP gradually gets worse. From the above analysis, it is known that because the fine aggregate segregation mixture has worse VSTP and LSDP, stress concentration occurs easily in a local area below the load range in mixture, and thus it is more likely to cause permanent deformation and form rutting. At the same time, it also can be seen from Figure 12 that there is no obvious correlation between the LSDP and the height of model.

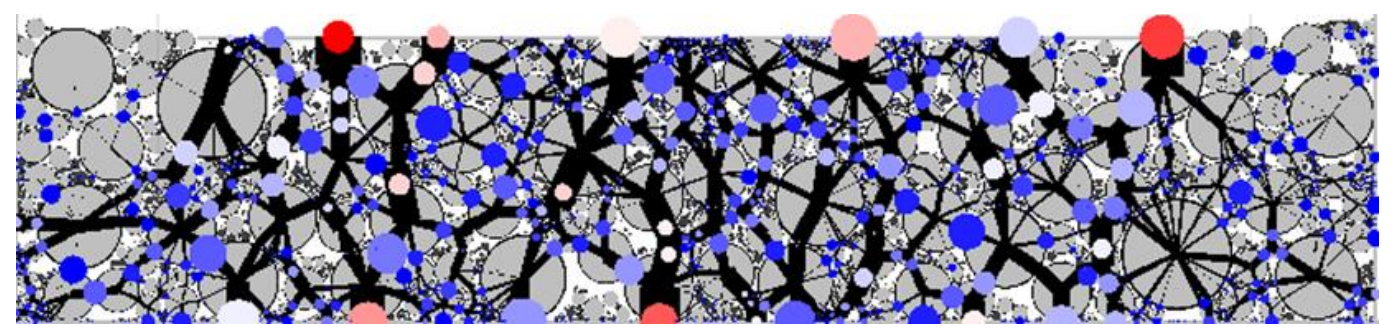

(a) $\mathrm{HC}$

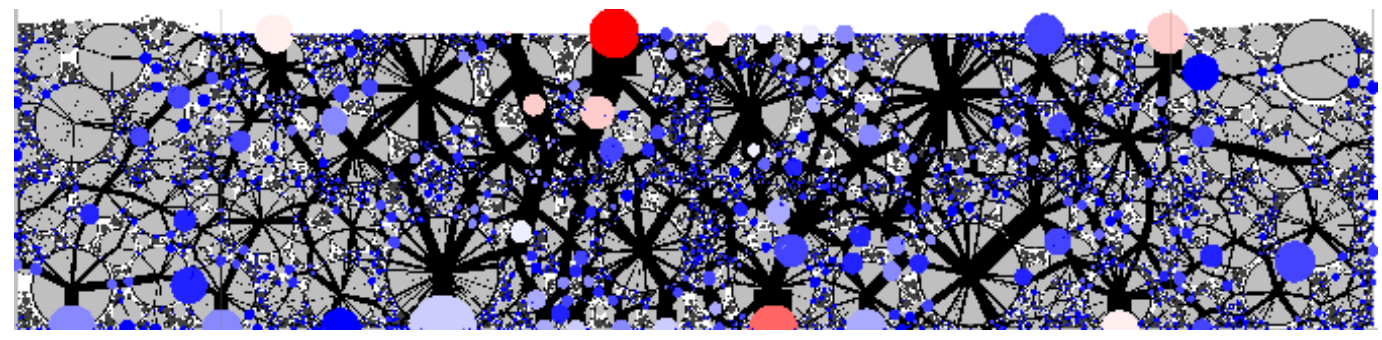

(b) $\mathrm{D}$

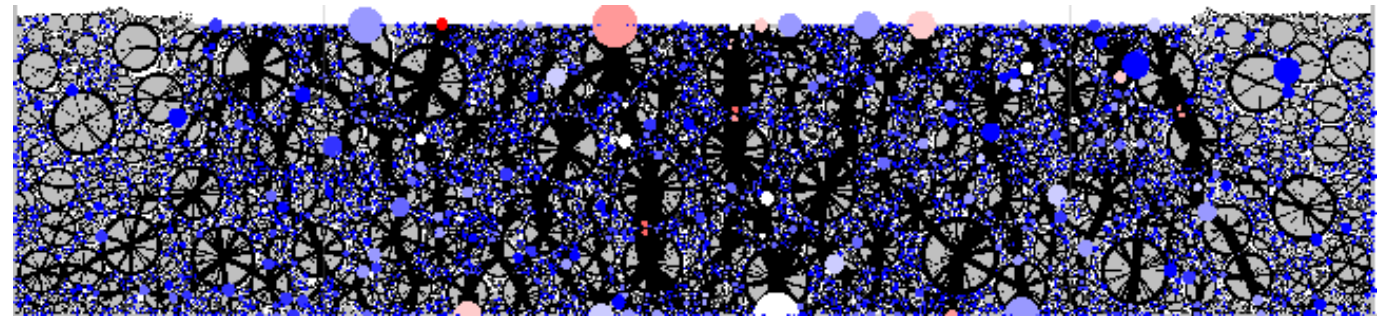

(c) $\mathrm{HF}$

Figure 11. Distribution of contact force and contact points in mixture skeletons.

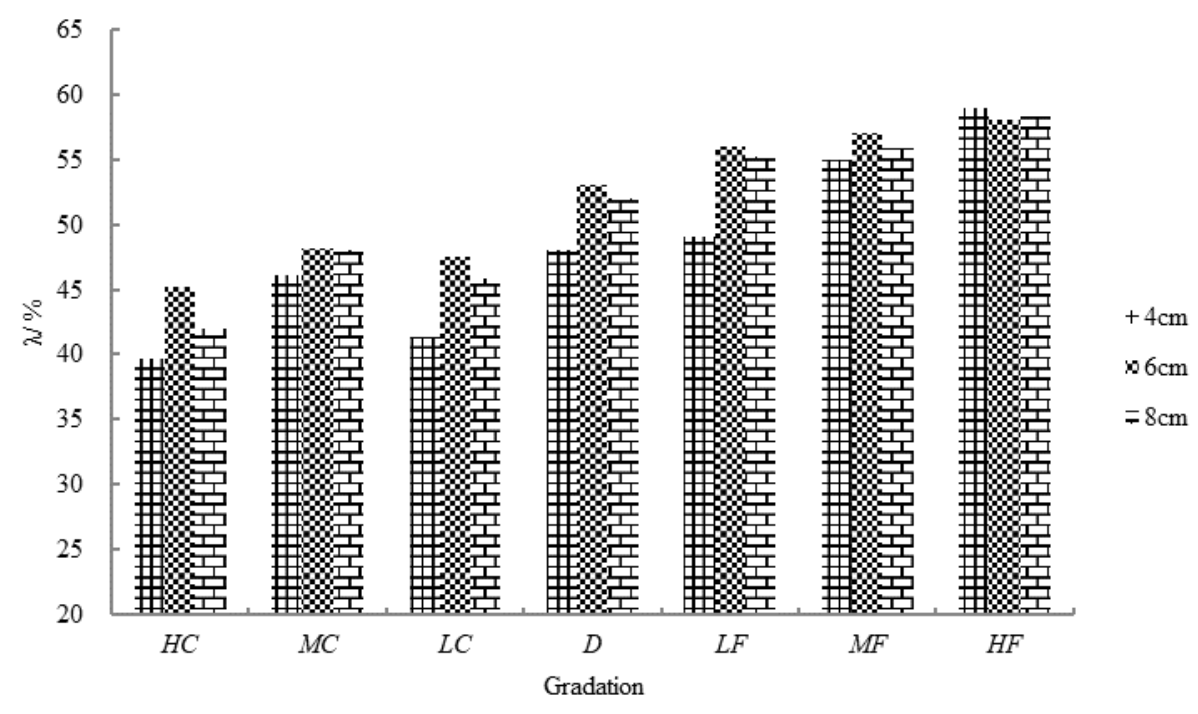

Figure 12. $\lambda$ value of asphalt mixtures. 
In addition, to further explore the contribution rate of aggregate with different diameters under load, the average contact forces around the aggregates were calculated. The contribution rate was then calculated using Equations (4) and (5). The result is shown in Figure 13.

$$
\begin{gathered}
\bar{F}=\frac{\sum_{j=1}^{N_{i}} F_{i, j}}{N_{i}} \\
\eta_{i}=\frac{\bar{F}_{i}}{\sum_{i=1}^{S} \bar{F}_{i}} \times 100 \%
\end{gathered}
$$

where,

$\bar{F}_{i}=$ average contact force of the $i$-th group of aggregate;

$F_{i, j}=$ normal contact force at the $j$-th contact point of the $i$-th group of aggregate;

$N_{i}=$ number of all contact points of the $i$-th group of aggregate;

$\eta_{i}=$ contribution rate to resist load of the $i$-th group of aggregate, and

$S=$ number of group divided by particle size in mixture.

As shown in Figure 13, the contribution rate to resist compressive load of the skeletal structure composed of aggregates whose particle size are above $2.36 \mathrm{~mm}$ is more than $70 \%$. In addition, the gradation segregation will affect the contribution rate of aggregates with different particle sizes. With the gradation from coarse to fine, the contribution rate of the $>13.2 \mathrm{~mm}$ grade aggregate gradually decreases, while that of the $<2.36 \mathrm{~mm}$ grade aggregate gradually increases. This indicates that with the increase of fine aggregates, the internal skeleton structure of the mixture changes, the number of fine aggregates participating in resisting the compression load increases gradually, and the resistance to load of the fine aggregates gradually intensifies. Moreover, with the gradation from coarse to fine, the contribution rate of each group of aggregate tends to be close, indicating that the distribution of contact force is more and more uniform in the mixture.

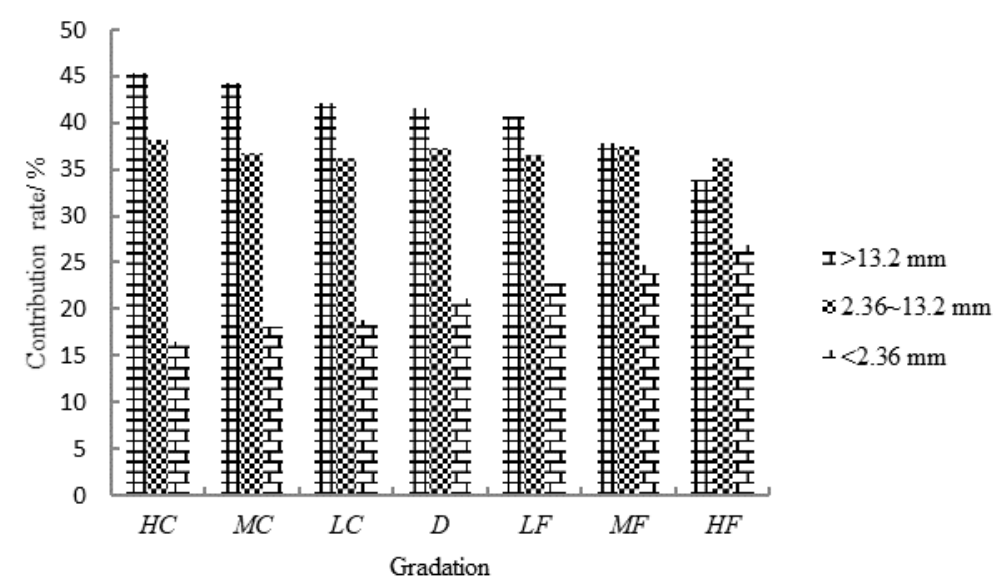

Figure 13. Contribution rate to load resistance for aggregates with different particle sizes.

\section{Conclusions}

In this study, 2D DEM models were developed for asphalt mixtures with different gradation segregation levels. Based on these models, the influences of gradation segregation on the structural characteristics and mechanical properties of asphalt mixture skeleton were studied. The main conclusions can be drawn from the above: 
(1) The aggregates skeleton of the asphalt mixture is mainly composed of coarse aggregates which are in contact with each other and whose particle sizes are greater than a certain value, while the fine aggregates mainly play the role of filling. The gradation segregation affects the constitution of the aggregate skeleton.

(2) With the gradation from coarse to fine, the VMA gradually decreases, the number of contact points between aggregates increases sharply, and the average contact force and the maximum contact force on the contact points gradually decrease.

(3) With the gradation from coarse to fine, the ability of the aggregate skeleton to resist a load gradually increases, and the segregation of coarse aggregates has a more significant effect on load resistance; the VSTP of the mixture skeleton gradually decreases, and the influence of segregation of coarse aggregate on it is larger; the LSDP of the mixture skeleton gradually weakens, and there is no obvious correlation between the LSDP and the degree of segregation when the segregation of coarse aggregate occurs, but when the segregation of fine aggregate occurs, the LSDP increases with the degree of segregation intensifying.

(4) The VSTP of the mixture skeleton gradually decreases with the increase of model height, and there is no obvious correlation between the LSDP and the model height.

(5) The contribution rate to resist the compressive load of the skeletal structure composed of aggregates whose particle size is above $2.36 \mathrm{~mm}$ is more than $70 \%$. With the gradation from coarse to fine, the contribution rate of coarser aggregates decreases and that of finer aggregates increases. The contribution of each group of aggregate tends to be balanced.

Author Contributions: Conceptualization, W.W. and Z.T.; methodology, Z.Z. (Zihan Zhu); software, W.W.; validation, W.W., Z.T. and Y.L.; formal analysis, Z.T.; investigation, W.W.; resources, W.W.; data curation, Z.T.; writing-original draft preparation, Z.T. and Z.Z. (Zeyu Zhang); writing-review and editing, Z.Z. (Zeyu Zhang); visualization, W.W.; supervision, W.W.; project administration, W.W.; funding acquisition, Y.L."

Funding: This research was funded by the National Natural Science Foundation of China, grant number 51008131 and 51038004 .

Conflicts of Interest: The authors declare no conflict of interest.

\section{References}

1. Stroup-Gardiner, M.; Brown, E.R. Segregation in Hot-Mix Asphalt Pavements; Transportation Research Board: Washington, DC, USA, 2000.

2. Leng, Z.; Zhang, Z.; Zhang, Y.; Wang, Y.; Yu, H.; Ling, T. Laboratory evaluation of electromagnetic density gauges for hot-mix asphalt mixture density measurement. Constr. Build. Mater. 2018, 158, 1055-1064. [CrossRef]

3. Stroup-Gardiner, M. Influence of segregation on pavement performance. In Proceedings of the Association of Asphalt Paving Technologists, Saint Paul, MN, USA, 2000.

4. Leng, Z.; Al-Qadi, I.L.; Lahouar, S. Development and validation for in situ asphalt mixture density prediction models. NDT E Int. 2011, 44, 369-375. [CrossRef]

5. Lv, S.; Liu, C.; Yao, H.; Zheng, J. Comparisons of synchronous measurement methods on various moduli of asphalt mixtures. Constr. Build. Mater. 2018, 158, 1035-1045. [CrossRef]

6. Lv, S.; Liu, C.; Chen, D.; Zheng, J.; You, Z.; You, L. Normalization of fatigue characteristics for asphalt mixtures under different stress states. Constr. Build. Mater. 2018, 177, 33-42. [CrossRef]

7. Lv, S.; Liu, C.; Lan, J.; Zhang, H.; Zheng, J.; You, Z. Fatigue Equation of Cement-Treated Aggregate Base Materials under a True Stress Ratio. Appl. Sci. 2018, 8, 691. [CrossRef]

8. Yu, J.M.; Yu, X.S.; Gao, Z.M.; Guo, F.; Wang, D.Y.; Yu, H.Y. Fatigue Resistance Characterization of Warm Asphalt Rubber by Multiple Approaches. Appl. Sci. 2018, 8, 1495. [CrossRef]

9. Bruno, L.; Parla, G.; Celauro, C. Image analysis for detecting aggregate gradation in asphalt mixture from planar images. Constr. Build. Mater. 2012, 28, 21-30. [CrossRef]

10. Jiang, W.; Li, Z.; Zhang, X. Effect of Gradation Segregation on Resistance of Moisture-induced Damage of Asphalt Mixture. J. Highw. Transport. Res. Dev. 2010, 36, 12-16. 
11. Guo, D.; Sha, A.M.; Sun, J.H.; Zhou, W.H.; Yang, X.M. Research on the Application of Infrared Thermal Camera in Asphalt Pavement Segregation Analysis. J. Highw. Transp. Res. Dev. 2007, 24, 63-66.

12. Liu, T.; Zhang, X.N.; Li, Z.; Chen, Z.Q. Research on the homogeneity of asphalt pavement quality using X-ray computed tomography (CT) and fractal theory. Constr. Build. Mater. 2014, 68, 587-598. [CrossRef]

13. Hassan, N.A.; Airey, G.D.; Khan, R.; Collop, A.C. Nondestructive characterisation of the effect of asphalt mixture compaction on aggregate orientation and segregation using X-ray computed tomography. Int. J. Pavement Res. Technol. 2012, 5, 84-92.

14. Masad, E.; Olcott, D.; White, T.; Tashman, L. Correlation of fine aggregate imaging shape indices with asphalt mixture performance. Geomaterials 2001, 1757, 148-156. [CrossRef]

15. Ling, M.; Luo, X.; Hu, S.; Gu, F.; Lytton, R.L. Numerical Modeling and Artificial Neural Network for Predicting J-Integral of Top-Down Cracking in Asphalt Pavement. Transp. Res. Rec. 2017, 2631, 83-95. [CrossRef]

16. Mahoney, J.P.; Muench, S.T.; Pierce, L.M.; Read, S.A.; Jakob, H.; Moore, R. Construction-related temperature differentials in asphalt concrete pavement-Identification and assessment. Transp. Res. Rec. 2000, 1712, 93-100. [CrossRef]

17. Sivilevicius, H.; Vislavicius, K. Stochastic simulation of the influence of variation of mineral material grading and dose weight on the homogeneity of hot-mix asphalt. Constr. Build. Mater. 2008, 22, 2007-2014. [CrossRef]

18. Bessa, I.S.; Branco, V.T.; Soares, J.B.; Neto, J.A. Aggregate shape properties and their influence on the behavior of hot-mix asphalt. J. Mater. Civ. Eng. 2014, 27, 04014212. [CrossRef]

19. Yu, H.Y.; Leng, Z.; Zhou, Z.Y.; Shih, K.M.; Xiao, F.P.; Gao, Z.M. Optimization of preparation procedure of liquid warm mix additive modified asphalt rubber. J. Clean. Prod. 2017, 141, 336-345. [CrossRef]

20. Zelelew, H.M.; Papagiannakis, A.T. Micromechanical Modeling of Asphalt Concrete Uniaxial Creep Using the Discrete Element Method. Road Mater. Pavement Des. 2010, 11, 613-632. [CrossRef]

21. Coenen, A.R.; Kutay, M.E.; Sefidmazgi, N.R.; Bahia, H.U. Aggregate structure characterisation of asphalt mixtures using two-dimensional image analysis. Road Mater. Pavement Des. 2012, 13, 433-454. [CrossRef]

22. Wagoner, M.P.; Buttlar, W.G.; Paulino, G.H. Development of a single-edge notched beam test for asphalt concrete mixtures. J. Test. Eval. 2005, 33, 452-460.

23. Masad, E.; Tashman, L.; Somedavan, N.; Little, D. Micromechanics-based analysis of stiffness anisotropy in asphalt mixtures. J. Mater. Civ. Eng. 2002, 14, 374-383. [CrossRef]

24. Li, X.; Chen, S.; Xiong, K.; Liu, X. Gradation Segregation Analysis of Warm Mix Asphalt Mixture. J. Mater. Civ. Eng. 2018, 30, 04018027. [CrossRef]

25. Yu, H.; Shen, S.J.C. Impact of aggregate packing on dynamic modulus of hot mix asphalt mixtures using three-dimensional discrete element method. Constr. Build. Mater. 2012, 26, 302-309. [CrossRef]

26. Zhang, Y.; Leng, Z.; Dong, Z.J.; Liu, Z.Y.; Zhang, Z.Y.; Tan, Z.F. Performance verification of various bulk density measurement methods for open- and gap-graded asphalt mixtures using $X$-ray computed tomography. Constr. Build. Mater. 2018, 158, 855-863. [CrossRef]

27. Zhang, Y.; Leng, Z. Quantification of bituminous mortar ageing and its application in ravelling evaluation of porous asphalt wearing courses. Mater. Des. 2017, 119, 1-11. [CrossRef]

(C) 2019 by the authors. Licensee MDPI, Basel, Switzerland. This article is an open access article distributed under the terms and conditions of the Creative Commons Attribution (CC BY) license (http://creativecommons.org/licenses/by/4.0/). 